\title{
Instituciones de enseñanza superior de España y Brasil: temática sobre las personas con discapacidad
}

CDD. 20.ed. 375

796.019

http://dx.doi.org/10.1590/1807-55092015000200303

\author{
Marina Brasiliano SALERNO* \\ Miguel Angel TORRALBA JORDAN** \\ Paulo Ferreira de ARAÚJO****
}

*Centro de Ciências
Humanas e Sociais,
Universidade Federal
de Mato Grosso do Sul.
**Facultad de Educa-
ción, Universitat de
Barcelona - Espanha.
***Faculdade de Edu-
cação Física, Univer-
sidade Estadual de
Campinas.

Las discusiones acerca de la población con discapacidades fueron insertadas en los cursos de Ciencias de la Actividad Física y Deporte y Educación Física como forma de atender a las disposiciones acordadas en diferentes documentos legales elaborados a lo largo de discusiones sobre el proceso de inclusión. Este proceso resultó en una mayor participación de ese grupo en otros ámbitos sociales entre ellos el deporte, el ocio y las competiciones. Eso nos llevó a preguntarnos cómo esta temática es abordada en los cursos citados. Para comprender este factor analizamos los planes docentes o programas de asignaturas de dos instituciones, una de Brasil y otra de España, que ofrecen estos cursos y que se destacan por la relevancia en el área en sus países. Observamos que la cantidad de horas de trabajo dedicado a la discusión sobre las personas con discapacidad es prácticamente la misma en los dos paises, aunque la institución brasileña ofrezca mayor cantidad de asignaturas. Además notamos que la institución brasileña presenta repeticiones de temas que tratan especificamente de la etiología de la discapacidad. Las lecturas especificas indicadas en los programas pueden ser más diversificadas en algunas asignaturas. Por fin, notamos que apenas las asignaturas con temática especifica sobre las personas con discapacidad las abordan. Creemos que los cursos pueden ofrecer más posibilidades de discusión sobre esta población en otras asignaturas, ya que las personas con discapacidad participan activamente de diversos espacios donde los profesionales de la Educación Física actúan.

PalavRAS-Chave: Discapacidad; Formación; Currículum; Inclusión.

\section{Introducción}

Este artículo forma parte de una investigación de doctorado realizada en Brasil y en colaboración con una institución de enseñanza superior de España, donde se buscó comprender, en ese primer momento, la temática acerca de las personas con discapacidad presente en los cursos de formación en Educación Física y Ciencia de la Actividad Física y Deporte.

Para realizarla, elegimos una institución de cada país con gran representatividad e influencia en el área. Buscamos comprender la organización de los cursos y analizamos los planes docentes o programas de las asignaturas que estaban disponibles en la página oficial de las instituciones, sus contenidos e indicaciones de lecturas, así, encontramos asignaturas obligatorias, específicas sobre la población con discapacidad y otros componentes, como las prácticas con supervisión de los profesores, momento que puede proporcionar el contacto entre los estudiantes y ese grupo.

Algunas Universidades ofrecen, aún, proyectos para las personas con discapacidades en los cuales aquellos alumnos que tengan interés pueden participar y estrechar el contacto con esa población. Este espacio, con todo, no fue el objeto de nuestra investigación.

Sabemos, todavía, que los cursos del grado en Educación Física son el primer momento de la formación específica en el área y con la autonomía de las Universidades la configuración de los cursos puede variarse, así como las posibilidades de formación y formación continuada, con la diferenciación entre los países de acuerdo con sus políticas de formación del profesorado ${ }^{1}$.

Aunque sepamos que existen otros momentos en la formación profesional elegimos el grado por 
ser, para muchos, el primer contacto con el área de la Educación Física en una perspectiva diferente de los practicantes de actividad física.

La elección de estos dos países fue debido a la semejanza existente entre ellos cuando analizamos las diferentes influencias que el área tuvo a lo largo de su trayectoria: del militarismo, del uso del área como forma de fortalecer al nacionalismo, de la fuerza de los ideales higienistas, entre otros aspectos ${ }^{2-6}$.

Creemos que todos eses factores tienen influencia en la consolidación del área en la enseñanza superior, ya que los objetivos, contenidos y estrategias son elementos determinados por el contexto e ideologías de la época. Así, el carácter biológico de la Educación Física puede ser considerado como predominante por muchos años, hecho que, en conjunto con otros pensamientos, mantuvo el carácter excluyente de la Educación Física.

Con el hecho del Año Internacional de los Impedidos, en 1981, declarado por las Naciones Unidas, los documentos internacionales elaborados después de esa fecha como forma de alcanzar los

\section{Método}

En ese estudio elegimos dos instituciones de enseñanza superior con gran prestigio y representatividad en Cataluña (España) y otra en São Paulo (Brasil). La primera ofrece el curso de Ciencia de la Actividad Física y Deporte y la segunda el curso de Educación Física.

Para alcanzar nuestro objetivo y comprender la temática abordada sobre las personas con discapacidad en ese primer momento de la formación profesional buscamos analizar los planes docentes o programas de las asignaturas disponibles en la página oficial de las instituciones. Los datos presentados fueron cogidos en el primer semestre de 2013.

\section{Resultados y discusiones}

Para comprender la organización de los dos centros de enseñanza, presentamos un primer análisis general, con el total de los créditos que deben ser cursados por los estudiantes.

El primer curso analizado es el de Brasil. Esta facultad pertenece a una universidad de referencia y recibe estudiantes de todo el país, ofreciendo dos períodos de estudios: el curso integral (donde retos trazados, como la Declaración de Salamanca ${ }^{7}$, ayudaron en el proceso de inclusión de las personas con discapacidad en los diferentes ámbitos sociales.

Tales documentos influenciaron también la formación de los profesionales en Educación Física de ambos países estudiados, ya que en los acuerdos constaba la necesidad de acciones gubernamentales, hecho que ha alcanzado la enseñanza superior. Podemos citar, en Brasil, la Resolución 03/87 que hace incluir en el currículo de los cursos de Educación Física asignaturas que abarquen discusiones sobre las personas con discapacidad ${ }^{8-9}$, hecho reforzado por las Resoluciones 1 de 2002 del Consejo Nacional de Educación $(\mathrm{CNE})^{10}$, con la normativa para la formación en las licenciaturas y 7 de 2004 del $\mathrm{CNE}^{11}$ que ofreció los indicativos para la reorganización de la formación en Educación Física. En España, el Real Decreto 1670/1993 establece que, inserida en la materia de actividad física y salud, se debe encontrar la actividad física para poblaciones especiales, entre ellas, comprendiese las personas con discapacidad ${ }^{12-13}$.

Esa investigación se caracterizó como cualitativa de cuńo interpretativo y documental, enfocando el análisis de los contenidos presentes en los documentos y la secuencia a ellos atribuida. Los parámetros utilizados para el análisis fueron: el objetivo, la carga horária de las signaturas, las propuestas de lecturas y el contenido elegido. A partir de estos datos, alisamos los indicativos de la configuración de las signaturas y la tendencia demostrada por los maestros responsables por esas discusiones a lo largo del grado.

Para contextualizar los cursos iniciamos nuestro análisis por la trayectoria presentada en las páginas oficiales de las instituciones y contrastadas con la información facilitada por el personal del centro.

las asignaturas son desarrolladas por las mañanas y tardes) con duración de cuatro años y el curso nocturno con duración de cinco años.

En esta facultad hay dos posibilidades de formación: la licenciatura, que ofrece la formación necesaria para que los profesionales impartan clases en la educación formal o no formal; y el grado en educación física que permite que los profesionales 
trabajen con el deporte, el ocio y la actividad física, en centros que no sean educacionales.

Notamos pequeña diferenciación de los créditos ( 1 crédito $=15$ horas) que deben ser cursados para obtención de la titulación: licenciatura del curso integral con 220 créditos (3300 horas); grado del curso integral con 216 créditos (3240 horas); licenciatura del curso nocturno con 218 créditos (3270 horas); grado en el curso nocturno con 214 créditos (3210 horas).

La formación empieza con el ofrecimiento de un núcleo común, con asignaturas hechas por todos los estudiantes que al final del segundo curso optan por seguir con la licenciatura o con el grado, siendo posible hacer los dos permaneciendo uno o dos años más. Esa organización de la Universidad es diferente de otras que ya ofrecen la prueba de acceso a la Universidad específica para la licenciatura o para el grado, o sea, los estudiantes necesitan escoger antes del ingreso en la facultad cuál formación desea hacer, hecho que no pasa con los estudiantes de la institución analizada, donde los candidatos hacen una selectiva y después de dos años hacen la elección de la titulación.

Algunas materias son compartidas con otras facultades como, por ejemplo, las asignaturas de anatomía, fisiología y bioquímica que son cursadas en la Facultad de Biología; las específicas de la educación (psicología de la educación, prácticas en la escuela y otras) son impartidas por docentes de la Facultad de Educación, por lo que los estudiantes pasan obligatoriamente por otras facultades.

Cuando analizamos la distribución de los créditos, notamos que la diferencia entre los cursos ofrecidos está en la obligatoriedad para los cursos integrales en cursar dos créditos más en optativas.

Las optativas se dividen en específicas y no específicas. Las primeras son aquellas que versan sobre la pedagogía del deporte y de la actividad física o para profundizar en una actividad física o deporte.

Las optativas no específicas componen un número de créditos que los estudiantes de ambas formaciones pueden elegir en cualquier curso ofrecido en la Universidad, o sea, los estudiantes pueden matricularse en clases de la Facultad de Educación, de Economía, de Biología, de Física, de Medicina, de Arte u otra cualquiera, respetando, claro, la organización y requisito de cada asignatura. Son eses créditos que deben ser cumplidos a más por los estudiantes del curso integral, como se ha dicho anteriormente.

Cuando analizamos las asignaturas que versan específicamente sobre las personas con discapacidad encontramos una en el núcleo común, una en la licenciatura y una asignatura en el grado, cada una de ellas con cuatro créditos. Eso significa decir que un estudiante que escoja hacer apenas una formación hará ocho créditos destinados a la discusión sobre las personas con discapacidad, lo equivalente a 120 horas. Ya aquellos que escojan hacer las dos formaciones cursaran tres asignaturas: 12 créditos, 180 horas.

La elección de la Facultad de Educación Física en Brasil fue hecha por la relevancia para el área, en España seguimos el mismo indicador, la referencia que la institución tiene en el país.

En la facultad de España, analizamos la formación específica en el área de la actividad física. Así, encontramos la primera diferenciación en la nomenclatura utilizada, ya que los cursos son de grado en Ciencia de la Actividad Física y el Deporte. Para obtención del título, los estudiantes deben cursar 240 créditos ECTS (European Credit Transfer System) que cumplen con las normativas del sistema europeo de enseñanza superior que objetiva la aproximación de la formación profesional en Europa ${ }^{14}$. Cada crédito ECTS equivale a 25 horas de trabajo presencial, dirigido y autónomo de los estudiantes. Así, la exigencia para la obtención del grado en Ciencia de la Actividad Física es de 6000 horas totales, con los estudios presenciales en las asignaturas y los estudios autónomos.

Eses créditos son divididos entre las asignaturas básicas (60 ECTS), obligatoria (150 ECTS), optativas (12 ECTS), prácticas externas (12 ECTS) y trabajo final de grado (6 ECTS).

Los estudiantes pueden escoger un itinerario específico en temas como la enseñanza, actividades en el medio natural, actividad física y salud, gestión deportiva o rendimiento deportivo. Con esa trayectoria, los estudiantes empiezan una especialización dentro de esa formación inicial, hecho que no es obligatorio en la facultad pero que puede auxiliar en el direccionamiento profesional.

Cuando buscamos las asignaturas que abarcan discusiones sobre la población con discapacidad encontramos una obligatoria a todos los alumnos (150 horas) y una optativa (60 horas).

Hasta ese punto, notamos la diferenciación de la nomenclatura de los cursos y la cantidad de horas de las dos instituciones de enseñanza superior. Algo interesante para ser observado es como en el espacio europeo de enseñanza, al cual España pertenece y se adecua a las normativas, da importancia a las horas de estudio fuera de la clase, o sea, el tiempo de estudio dedicado por los alumnos para hacer las propuestas de las aulas o profundizar los estudios son contabilizados en su formación, al contrario de la facultad en Brasil que enfoca el tiempo en la aula. 
Siguiendo esa línea, notamos que en Brasil el curso de Educación Física ofrece dos asignaturas obligatorias, considerando uno de los cursos (licenciatura o grado), y en España se ofrece una que es obligatoria y una optativa. En horas, en Brasil encontramos dedicación de 120 a 180 horas (dependiendo de la elección del estudiante por una o dos formaciones) y en España de 150 a 210 horas (cursando sólo la obligatoria o también la optativa). Cuando analizados los créditos totales de una formación brasileña y la formación española, el porcentaje de créditos destinados a la persona con discapacidad es prácticamente el mismo. La mayor diferenciación está en la forma de distribución de los créditos, ya que todas las horas en Brasil son dedicadas a las clases presenciales, y en cambio en España la asignatura contabiliza el estudio fuera del aula presencial. Sobre ese punto, necesitaríamos investigar las posibles consecuencias de esa distribución.

Después de comprender numéricamente como se organizan los dos centros, pasamos a los planes docentes o programas de las asignaturas que nos informan el objetivo, el contenido presentado, algunas estrategias utilizadas y la lectura sugerida.

De la facultad brasileña fueron analizados 55 programas de las asignaturas impartidas en la facultad. Las no analizadas son de otras facultades cuyas publicaciones no estaban disponibles para visualización o de la propia facultad de Educación Física las cuales constan en el currículo pero que no estaban siendo ofrecidas al momento de la cogida de los datos.

Entre las 55 signaturas presentes en los análisis, 35 pertenecen al núcleo común; cinco son específicas a la licenciatura y 15 al grado. Como hemos dicho anteriormente, tres asignaturas enfocan las discusiones sobre las personas con discapacidad, que a continuación analizamos:

\section{Educación física adaptada (núcleo común)}

Trabaja conceptos de la educación física adaptada, su histórico y consideraciones sobre los aspectos sociales. Caracteriza las discapacidades sensoriales, motrices y cognitivas.

El programa de la disciplina presenta sus objetivos con el foco puesto en los alumnos, que ellos puedan comprometerse con las posibilidades de la educación física, ampliando su mirada frente al fenómeno de la inclusión y su aplicación en los diferentes centros de actuación, dando especial atención a la escuela. Pretende que los alumnos puedan caracterizar las discapacidades apuntando posibilidades de trabajo con esa población, articulándose con otras áreas.

Los contenidos citados versan sobre los espacios dónde podemos encontrar la actividad física adaptada como terapia, deporte, rehabilitación o educación. A partir de esa introducción, pasa a dividir los contenidos por las discapacidades: física, intelectual, auditiva, visual, las síndromes y la parálisis cerebral. De cada una de estas presenta su característica, las etiologías y las posibilidades de actuación del profesional (actividades, metodologías, evaluaciones).

La bibliografía presenta nueve títulos que ayudan a los alumnos a profundizaren sus estudios en la asignatura con textos nacionales e internacionales. Cinco de ellos pueden ayudar en la caracterización del área de la educación física adaptada y de las discapacidades como por ejemplo los textos de WiNNICK $^{15}$ o MAUERBERG DE CASTRO ${ }^{16}$, tres presentan el enfoque en la evaluación de las habilidades motrices como de los autores Gorla y AraúJo ${ }^{17}$ y un específico a poblaciones con discapacidad intelectual ${ }^{18}$.

Algo que creemos que hace falta en las referencias, debido a la especial atención dada a la escuela en los objetivos de la asignatura, son más fuentes de lecturas que direccionen para esa institución. Aunque sea la primera asignatura a la cual los alumnos tendrán contacto acerca de esa población y no sea específica a la licenciatura, como el documento cita la escuela es interesante que se pueda presentar a los estudiantes indicativos para realizar lecturas específicas.

\section{Educación física escolar especial (licenciatura)}

Objetiva reflexionar sobre los conceptos y procedimientos pedagógicos de la educación física escolar especial.

El contenido empieza con la reflexión de la trayectoria del proceso de inclusión de las personas con discapacidad en las escuelas regulares y la educación física frente a esa modificación.

Siguen los contenidos dividiendo las discapacidades entre visuales, físicas, auditivas e intelectuales y presentándolas de forma teórica y práctica. Percibimos que hace uso de muchos recursos visuales y busca que los estudiantes piensen en planes de enseñanza para grupos en los cuales encuentran alumnos con discapacidad.

La bibliografía que auxilia a los alumnos a profundizaren sus lecturas y reflexiones presenta 17 títulos desde libros hasta trabajos de conclusión de grado. Observamos que la mayoría de los textos enfocan la temática de la educación física e inclusión de alumnos 
con discapacidades, con 10 títulos, como ejemplo citamos Rodrigues ${ }^{19}$ y Silva et al. ${ }^{9}$. Tres versan sobre la construcción de la inclusión, sus influencias y proceso de comprensión y atención a las personas con discapacidades en Brasil, como el texto de Silva et al. ${ }^{20}$. Otros cuatro textos discuten posibilidades de prácticas pedagógicas de la educación física, no necesariamente relacionando a los alumnos con discapacidades, pero ofrecen una mirada diferente a la educación física escolar para que todos los alumnos puedan participar efectivamente, como en las reflexiones propuestas por DAÓLIO ${ }^{21}$ y PICCOLO ${ }^{22}$.

Nos pareció que hay la duplicación del contenido explorando las discapacidades teórica y prácticamente, como observado en la asignatura anterior. Ese momento podría dedicarse a discurrir sobre la diversidad en la escuela, cómo aborda la, cómo estimular la interacción entre alumnos con y sin discapacidades.

Siendo esa disciplina específica de la licenciatura, elementos específicos de la escuela y de la educación física en eso espacio deben, en nuestra comprensión, presentaren se más enfáticamente.

\section{Deporte adaptado (grado)}

Trata de presentar las diferentes modalidades deportivas que son adaptadas o fueron creadas para las personas con discapacidad.

El contenido es fundamentalmente las reglas y formas de organización del deporte paralímpico. Hay espacio para reflexionar sobre el deporte escolar, la organización de competiciones y deportes en la naturaleza. Las modalidades citadas son: goalball, voleibol sentado, atletismo, natación, luchas, baloncesto, rugby sobre silla de ruedas, fútbol de $5 \mathrm{y}$ 7 y ciclismo. Aún ofrecen una atención especial a las personas con discapacidad intelectual.

Presenta 10 fuentes en la literatura, incluyendo libros, investigaciones de masteres y tesis doctorales para que los alumnos puedan buscar más basamento teórico y dos enlaces de los Comités Paralímpicos, internacional y brasileño.

Analizando las indicaciones de lectura, notamos la predominancia de asuntos relacionados con las personas con discapacidad visual, observando que de los 10 textos utilizados en las referencias, siete abordan exclusivamente esa población. Dos traen informaciones de otras discapacidades, como el texto de Gorgatti y $\operatorname{Costa}^{23}$ y uno sobre el deporte escolar.

Eso puede ser comprendido como algo contradictorio de la asignatura, ya que apunta modalidades que son practicadas por personas con discapacidades motrices, por ejemplo, pero indica poca literatura sobre ellas.

Pensando en la intención de comprender aún la trayectoria del deporte adaptado, no podemos dejar de citar textos sobre su construcción y consolidación en Brasil ${ }^{24-25}$, que tendría su continuación con el texto presentado en el plan docente ${ }^{26}$ que empieza a mostrar las posibilidades de trabajo efectivo con personas con discapacidad, en eso caso específicamente con las visuales.

A partir de ese análisis, comprendemos que tanto los alumnos de la licenciatura como del grado pueden complementar sus conocimientos con las dos asignaturas que son obligatorias en los cursos.

Sin embargo, notamos en la licenciatura una repetición del contenido teórico sobre las discapacidades, que ya son abordados en la asignatura del núcleo común y que podrían ser clases utilizadas para tratar o profundizar otros contenidos.

Creemos, todavía, que el contenido de la asignatura del núcleo común también pueda ser alterado ya que los alumnos pueden estudiar sobre las discapacidades en otra asignatura: fundamentos neurofuncionales ${ }^{1}$ del movimiento, que presenta los diferentes sistemas y órganos con sus funcionamientos, correctos o no. De esa forma, otros temas podrían ser abordados y profundizados.

Otro elemento que nos llamó la atención fueron las referencias utilizadas en la asignatura del grado que enfoca más las personas con discapacidad visual, pero en los contenidos amplían las posibilidades de trabajo para otras etiologías de discapacidad. Eso, con todo, puede ser reflejo del interés del profesor, ya que el contenido de los programas de las signaturas también son condecentes con el interés profesional del maestro.

Esclarecemos aquí que comprendemos que los textos pueden ser complementados a lo largo del trabajo con la asignatura, con todo, vemos que hay una laguna que necesita ser percibida y superada siendo el programa de la disciplina un documento de referencia para los estudiantes.

Otro elemento que se destaca es la preocupación observada con la escuela en los tres programas de las asignaturas, respetando sus especificidades. La primera lleva la escuela, nos pareció, en las discusiones de forma más general; la específica de la licenciatura profundizando algunas cuestiones sobre la escuela; y la del grado destacando el deporte escolar. Ese hecho resalta que ese espacio de enseñanza presenta su relevancia en la participación de las personas con discapacidades y debe presentarse en la formación en Educação Física de forma a profundizar las 
discusiones contribuyendo con el profesional que actuará en este espacio.

A partir de los programas de las asignaturas específicas sobre las personas con discapacidad observamos que los estudiantes que escogen las dos formaciones podrán desarrollar una compresión más completa, ya que la temática abordada abarca el proceso de inclusión, el deporte adaptado y la inclusión en la escuela. Eso es puesto a partir del análisis de los planes docentes, que pueden ser complementados a lo largo del ofrecimiento de la asignatura y a partir de la curiosidad de los estudiantes.

Analizando los programas de las demás asignaturas, que no son directamente relacionadas a población con discapacidad, no encontramos mención directa a las personas con discapacidad en los objetivos o en los contenidos presentados.

Con todo, en la asignatura de Lucha encontramos en la bibliografía un texto sobre la esgrima en silla de ruedas y el la Actividad Física para Grupos Diferenciados, que abarca personas con diferentes enfermedades como patologías cardíacas, diabetes, hipertensión arterial, obesidad, presenta, también en su orientación para lecturas, un texto que aborda la persona con necesidades especiales.

Hay, aún, otras disciplinas que tratan de factores que determinan patrones y que pueden mencionar los que están fuera de ellos, como la disciplina que trata del desarrollo motriz, fundamentos neurológicos o crecimiento y desarrollo, pero no mencionan nada específico a la población con discapacidad.

En aquellas que tratan de los deportes tampoco encontramos mención a las personas con discapacidad, aunque sean las pedagógicas, que son cursadas también por los estudiantes de licenciatura que van a trabajar con las actividades en grupos heterogéneos donde pueden encontrarse con alumnos con discapacidad. Eso, con todo, no es explorado por los docentes de esas asignaturas, según los análisis de los programas.

Eso nos muestra que, a partir del análisis de los programas de las asignaturas, prácticamente todas las posibilidades de discusión sobre las personas con discapacidad están depositadas en las asignaturas específicas. Claro que comprendemos que las clases son siempre dinámicas y que los asuntos pueden ser modificados con la trayectoria de las mismas, todavía, en un primer momento de reflexión sobre los contenidos y bibliografías, la población con discapacidad no fue englobada en las demás asignaturas, con excepción de la de lucha y actividad física para grupos diferenciados que han hecho una citación en las referencias bibliográficas, pero comprendemos la limitación de ese análisis ya que no tuvimos acceso a todos los textos que componen la bibliografía de cada asignatura y no siempre el título encierra el contenido del artículo.

Siguiendo con nuestro análisis, nos introducimos en el curso de Grado en Ciencia de la Actividad Física y Deporte de la institución de enseñanza superior elegida en España.

En la página web de la facultad encontramos disponibles 56 planes docentes, siendo 35 que constan en el plan de estudios propuesto por la facultad a sus estudiantes y 21 de ampliación de la materia de deportes que ofrece posibilidades para la elección del estudiante de acuerdo con sus ansias.

Entre las asignaturas analizadas, describiremos la relacionada a las personas con discapacidad y apuntaremos otra que no tenía su plan docente disponible.

\section{Principios y bases de la actividade física y del deporto adaptado (obligatoria)}

Los objetivos trazados son ofrecer el conocimiento sobre el deporte adaptado, su desarrollo, perspectivas y posibilidades educativas, para que los estudiantes puedan adecuar su práctica en los diferentes ámbitos que son abarcados por la actividad física adaptada y la educación física. Eso se propone con la posibilidad de incremento de una actitud positiva frente a la inclusión de personas con discapacidad en la educación física escolar, en el deporte y otros.

Trabaja con el concepto de necesidades educativas especiales ofreciendo bloques temáticos sobre sus diferentes tipologías (física, intelectual, sensorial y otras) y la interconectividad entre ellos y la actividad física adaptada.

A partir de eso la asignatura propone analizar las posibilidades motrices para adecuar las tareas propuestas a las personas con necesidades educativas especiales, logrando enseńar los contenidos propios del área.

La bibliografía propuesta contiene nueve títulos nacionales e internacionales y dos leyes que sustentan las bases de la inclusión ${ }^{27-28}$. Observamos la tendencia de los textos que direccionan para la reflexión acerca de la relación entre profesores y alumnos con discapacidad, con cinco textos, de entre ellos el de GuTIÉRREZ et al. ${ }^{29}$ y más dos que abarcan temas sobre el proceso de inclusión, su trayectoria y necesidad de consolidación, como por ejemplo el texto de BLOCK y OBRUSNIKOVA ${ }^{30}$ que traen una revisión sobre la inclusión en el área de la Educación Física.

Además apunta cuatro títulos de revistas en castellano e inglés y dos enlaces para búsquedas sobre las posibilidades educativas y deportivas. 
En esa institución, encontramos una asignatura más que aborda específicamente el deporte, la actividad física y la educación física para personas con discapacidad y que está puesta en los itinerarios específicos de enseñanza y de actividad física y salud, con todo, no estaba disponible el plan docente.

En las asignaturas que no discuten específicamente temas sobre las personas con discapacidad no encontramos mención directa a esa población a lo largo de las descripciones de los contenidos y estrategias.

Así como en la institución brasileña, algunas asignaturas nos sugieren la posibilidad de abordaje del tema de la inclusión de personas con discapacidades ya que problematizan el desarrollo en la escuela.

En esa institución observamos, al contrario de la brasileña, la presentación en todos los planes docentes de las competencias que se proponen a desarrollar, tanto en general cuanto en la asignatura. Eso es interesante pues, aunque las dos instituciones ofrezcan los objetivos de formación del alumnado, en la española los encontramos con más transparencia y objetividad.

Es interesante pues las competencias generales siempre direccionan los estudiantes a conseguir solucionar problemas y lidiar con situaciones no previstas.

Con eso, se debe pensar que los estudiantes conseguirán utilizar todo el conocimiento obtenido a lo largo de su formación para adecuar su trabajo a cualquier población que a él se le presente, incluyendo a las personas con discapacidad.

Sobre ese punto ponderamos, todavía, que la formación no termina en el grado, las personas deben seguir estudiando y rellenando las lagunas que siempre se establecen sea en la formación inicial, por dudas y exigencias que emergen con nuestro cotidiano laboral o simplemente por la evolución de las áreas, para nuestra actualización. Sin embargo, pensamos en el grado como un momento en el que los alumnos se hallan abiertos a muchas informaciones y experiencias y presentarles la población con discapacidad en diferentes contextos puede facilitar la aproximación con ese grupo.

De acuerdo con la organización de los dos cursos de grado estudiados creemos posible que estos pueden ser mejor aprovechados cuando pensamos en la consolidación de la inclusión de las personas con discapacidad, pues actualmente ese grupo puede ser caracterizado en el entrenamiento de alto rendimiento, en la escuela, en las actividades en la naturaleza, en el ocio, en la iniciación deportiva, en las empresas, o sea, ellas están presentes en los diferentes sectores donde actúan los profesionales de educación física o ciencia de la actividad física.
Hablar de los grupos con discapacidades en diferentes asignaturas puede contribuir para que los estudiantes amplíen la mirada para la diversidad tan presente en todos los segmentos. Facilita la comprensión que todos son diferentes y que necesitamos conocerlos, así como a sus necesidades y capacidades, para que entonces el trabajo sea adecuado.

Sabemos que los planes docentes o programas de las asignaturas son documentos dinámicos, que son modificados a lo largo de su implementación y que son elaborados por los profesores que presentan interés en determinadas áreas y eso influencia las discusiones propuestas en esos momentos.

Todavía, es necesario pensar en qué los estudiantes necesitan para que sus actitudes y trabajo sean incluyentes, principalmente en la escuela, donde la diversidad está siempre presente. Observamos que la escuela es un espacio de destaque en la institución brasileña, con todo, las discusiones propuestas necesitan enfatizar ese espacio y sus características específicas. No es preciso apenas conocer las discapacidades, mas también conseguir superarlas y desarrollar clases donde todos puedan participar de acuerdo con el objetivo de la Educación Física en la escuela.

Así, creemos que el presentado en los planes docentes y programas de las asignaturas son contenidos relevantes para el área, con todo, las instituciones pueden revisarlos para incluir momentos efectivos de contacto directo con ese público, principalmente en la institución brasileña que nos pareció no ofrecer espacio de práctica, diferente de la institución de Cataluña que en su currículo, por adecuarlo al sistema Europeo de Enseńanza Superior, ofrece la disponibilidad de tiempo en la formación que incentivan al contacto entre los estudiantes y las personas con discapacidad.

Estudiar la formación profesional en Educación Física o Actividad Física y Deporte nos abre para reflexiones amplias. Siempre nos encontramos con discursos que afirman que los profesores en las escuelas deben recibir a todos los alumnos, que esos deben participar activamente, que debe haber profesionales capaces de trabajar con los diferentes deportes paralímpicos, pero la diversidad, a veces, parece estar en otro espacio que no en el de la formación inicial.

La población con discapacidad pasa a participar mucho más en los diferentes ámbitos sociales y su espacio está garantizado y consolidado. Todavía, aún nos vemos en un momento de transición, donde aún necesitamos de reflexiones y espacio para romper con barreras que todavía existen, y eso puede ser pensado en la formación a lo largo de la enseñanza superior. 
Comprendemos que las habilidades de los discentes deben llevarlos a conseguir analizar y adecuar sus prácticas relacionando los diferentes conocimientos adquiridos a lo largo del estudio de grado, pero el auxilio que puede ser recibido en una asignatura dará la posibilidad de profundizar el conocimiento sobre esa población, ya que las que tratan sobre las personas con discapacidad son pocas.

Actualmente no solo debemos direccionar el trabajo con esa población ya que esta se encuentra también en otros espacios más allá de la escuela, está en el deporte de alto rendimiento, busca actividades en el tiempo de ocio, está trabajando en los diferentes centros, hace diferentes actividades físicas. De esa forma, la formación en Educación Física o Ciencia de la Actividad Física no debe tratar las actividades para personas con discapacidad únicamente en las asignaturas específicas, pues estas están insertadas en la sociedad con la cual trabajamos.
Creemos que las asignaturas especificas son necesarias para que barreras aún existentes puedan ser superadas, pero que el contenido necesario para dar atención a esa población con calidad no cabe, solamente, dentro de estas.

Aunque las competencias esperadas de los discentes sean que los mismos consigan adecuarse a las situaciones, dando soluciones a los problemas, esa es una tarea que necesita ser construida juntamente con los docentes de la universidad que posean la experiencia para auxiliar en esas reflexiones. De esa forma, la articulación del conocimiento obtenido a lo largo de los estudios podrá darse de manera gradual e efectiva.

Notamos, así, con este análisis que hay muchas posibilidades insertadas en las asignaturas de los cursos de Educación Física y Ciencia de la Actividad Física que pueden ser usadas para ampliar la discusión sobre las personas con discapacidad, auxiliando en la efectuación de la inclusión de estas en el área de la Educación Física.

\begin{abstract}
Institutions of higher education in Spain and Brazil: topics on people with disabilities

Discussions about people with disabilities were inserted into the course of Physical Activity Sciences and Sports and Physical Education as a way to meet the agreed provisions in different legal documents developed over discussions about the inclusion process. This process resulted in a greater share of this group in other social areas including sport, leisure and competitions. That led us to ask how this issue is addressed at the listed courses. To understand this factor we analyzed teaching plans or programs of subjects from two institutions, one from Brazil and other from Spain, which offer these courses and highlights the relevance in the area at their countries. We noted that the number of hours of work devoted to the discussion of disability in the specific subjects is almost the same in both countries, although the Brazilian institution offers more subjects. We have also noticed that the Brazilian institution shows repetitions of topics that specifically address the etiology of the disability. Specific readings indicated in the programs can be more diversified at some subjects. Finally, we note that only the subjects with specific focus on people with disabilities address this population. We believe that courses may offer more possibilities for discussion on this in other subjects, because people with disabilities actively participate in many spaces where professionals of Physical Education act.
\end{abstract}

KEY WORDS: Disability; Graduation; Curriculum; Inclusion.

\title{
Referencias
}

1. Reid G. Preparação profissional em educação física adaptada: perspectivas norte americanas. Rev Sobama. 2000;1:1-4.

2. Castellani Filho L. Educação física no Brasil: a história que não se conta. Campinas: Papirus; 1994.

3. Soares CL. Educação física: raízes européias no Brasil. Campinas: Autores Associados; 1994.

4. Vega FD. La gimnasia en España: aportaciones de las Fuerzas Armadas. In: Blanco SG. Simposium de historia de la educación física. Salamanca: Universidad de Salamanca; 1995. p.11-6. 
5. Jordán ORC. Perspectivas y modelos en la formación inicial del profesorado de educación física en la educación primaria española. In: Blanco SG. Simposium de historia de la educación física. Salamanca: Universidad de Salamanca; 1996. p.31-41.

6. Pradillo JLP. El espacio profesional de la educación física en España: génesis y formación (1883-1961). Alcalá de Henares: Universidad de Alcalá; 1997.

7. UNESCO. Ministerio de Educación y Ciencia España. Declaración de Salamanca y marco de acción para las necesidades educativas especiales. Salamanca: Unesco; 1994. Disponible: http://www.unesco.org/education/pdf/SALAMA_S.PDF.

8. Brasil. Conselho Federal de Educação. Resolução CFE n. 3 de 16 de junho de 1987. Fixa os mínimos de conteúdo e duração a serem observados nos cursos de graduação em educação física (bacharelado e/ou licenciatura plena). Diário Oficial da União, Brasília (10 set. 1987). Disponible: http://www.ufpb.br/sods/consepe/resolu/1990/Res0387-cfe.htm.

9. Silva RF, Seabra Júnior L, Araújo PF. Educação física adaptada no Brasil: da história a inclusão educacional. São Paulo: Phorte; 2008.

10. Brasil. Conselho Nacional de Educação. Resoluçáo CNE/CP n.1 de 18 de fevereiro de 2002. Institui diretrizes curriculares nacionais para a formação de professores da educação básica, em nível superior, curso de licenciatura, de graduação plena. Disponible: http://portal.mec.gov.br/cne/arquivos/pdf/rcp01_02.pdf.

11. Brasil. Conselho Nacional de Educação. Resolução CNE/CES n.7 de 31 março de 2004. Institui as diretrizes xurriculares nacionais para os cursos de graduação em educação física, em nível superior de graduação plena. Oficial da União, Brasília (5 abr. 2004). Disponible: http://portal.mec.gov.br/cne/arquivos/pdf/ces0704edfisica.pdf.

12. Espańa. Real Decreto 1679 de 24 de septiembre de 1993. Establece el título univesitario oficial de licenciado en ciencias de la actividad física y del deporte y las directrizes generales propias de los planes de estudios conducentes a la obtención del mismo. Boletín Oficial del Estado, Madrid (20 oct. 1993);251:29558-9. Disponible: http://www.csd. gob.es/csd/estaticos/inst-dep/armonizacion-juridica/020.pdf.

13. Arroyo MPM, Vaíllo RR. Contexto institucional y curricular: el espacio europeo de educación superior. In: Vaíllo R R. La actividad física y deporte adaptado ante el espacio europeo de enseńanza superior. Sevilla: Wanceulen Editorial Deportiva; 2010. p.47-85.

14. España. Ministerio de Educación. Secretaria General de las Universidades. Guía del usuarios del ECTS. Bruselas: Secretaria General de las Universidades; 2009. Disponible: http://ec.europa.eu/education/lifelong-learning-policy/ doc/ects/guide_es.pdf.

15. Winnick JP. Educação física e esportes adaptados. Barueri: Manole; 2004.

16. Mauerberg de Castro E. Atividade física adaptada. Ribeirão Preto: Tecmed; 2005.

17. Gorla JI, Araújo PF. Avaliação motora em educação física adaptada. São Paulo: Phorte; 2007.

18. Eichstaedt CB, Lavay BW. Physical activity for individuals with mental retardation in infancy through adulthood. Champaign: Human Kinetics; 1992.

19. Rodrigues D. A educaçáo física perante a educação inclusiva: reflexôes conceptuais e metodológicas. R. Educ Fís/ UEM. 2003;14:67-73.

20. Silva RF, Araújo PF, Duarte E. Inclusão educacional: uma "roupa nova” para um “corpo velho”. Lect Educ Fis Deportes. 2004;10 Disponible: http://www.efdeportes.com/efd69/inclusao.htm.

21. Daólio J. Da cultura do corpo. Campinas: Papirus; 1995.

22. Piccolo VN. Educação física escolar: ser ... ou não ter? Campinas: UNICAMP; 1995.

23. Gorgatti MG, Costa RF. Atividade física adaptada. Barueri: Manole; 2005.

24. Araújo PF. Desporto adaptado no Brasil: origem, institucionalizaçáo e atualidade. Brasília: INDESP; 1998.

25. Araújo PF. Desporto adaptado no Brasil. São Paulo: Phorte; 2011.

26. Almeida JJG. Estratégias para aprendizagem esportiva: uma abordagem pedagógica da atividade motora para cegos e deficientes visuais [tese]. Campinas (SP): Universidade Estadual de Campinas; 1995.

27. España. Ley Orgánica 51/2003 de 2 diciembre de 2003. De la igualdad de oportunidades, no discriminación, y accesibilidad de las personas con necesidades educativas especiales. Boletín Oficial del Estado, Madrid (3 dic. 2003);289:43187-95.

28. España. Ley Orgánica 2/2006 de 3 mayo de 2006. De Educación. Boletín Oficial del Estado, Madrid (4 mayo 2006);106:17158-207.

29. Gutiérrez M, Pilsa C, Torres E. Perfil de la educación física y sus profesores desde el punto de vista de los alumnos. Rev Int Cienc Deporte. 2007;3:42-64.

30 Block ME, Obrusnikova I. Inclusion in physical education: a review of the literature from 1995-2005. Adapt Phys Activ Q. 2007;24:103-24. 
Salerno MB, et al.

\section{Agradecimentos}

A CAPES por el financiamiento del proyecto.

Marina Brasiliano Salerno

Curso de Educação Física

Centro de Ciências Humanas e Sociais

Universidade Federal de Mato Grosso do Sul

Cidade Universitária - Bloco IV - Caixa Postal 549

79070-900 - Campo Grande - MS - BRASIL

Revisado: 24/07/2014

Aceito: 12/09/2014

e-mail: marina.brasiliano@gmail.com

312 • Rev Bras Educ Fís Esporte, (São Paulo) 2015 Abr-Jun; 29(2):303-12 